MS25-O3 Atomic structure and phason modes of the Sc-Zn icosahedral quasicrystal

Marc de Boissieu ${ }^{1,2}$, Tusnetomo Yamada ${ }^{3}$, Hiroyuki Takakura ${ }^{4}$, Holger Euchner ${ }^{5}$, Cesar Pay-Gomez ${ }^{6}$, Alexey Bosak ${ }^{7}$, Pierre Fertey $^{8}$

1. Univ. Grenoble Alpes, SIMAP, F-38000, Grenoble, France

2. CNRS, SIMAP, F-38000, Grenoble, France

3. IMRAM, Tohoku University, Miyagi 980-8577, Japan

4. Division of Applied Physics, Faculty of Engineering, Hokkaido University, Hokkaido 060-8628, Japan,

5. Institute of Materials Science and Technology, Vienna University of Technology, Vienna 1040, Austria

6. Department of Chemistry, ${ }^{\circ}$ Angstr" om Laboratory, Uppsala University, Uppsala 75121 , Sweden

7. ESRF-The European Synchrotron, Grenoble F-38000, France,

8. Synchrotron SOLEIL, Gif-sur-Yvette F-91192, France,

email: marc.de-boissieu@simap.grenoble-inp.fr

The detailed atomic structure of the binary icosahedral $\mathrm{i}-\mathrm{ScZn}_{7,33}$ quasicrystal has been investigated by means of high-resolution synchrotron single-crystal Xray diffraction and absolute scale measurements of the diffuse scattering. The average atomic structure has been solved using the measured Bragg intensity data based on a six dimensional model that is isostructural to the $\mathrm{i}-\mathrm{YbCd}_{57}$ one. The structure is described with a quasiperiodic packing of large Tsai-type rhombic triacontahedron clusters and double Friauf polyhedra (DFP), both resulting from a close-packing of a large (Sc) and a small $(\mathrm{Zn})$ atom. The difference in chemical composition between $\mathrm{i}-\mathrm{ScZn}_{73}$ and $\mathrm{i}-\mathrm{YbCd}_{5}$ was found to lie in the icosahedron shell and the DFP where in $\mathrm{i}-\mathrm{ScZn}_{733}$ chemical disorder occurs on the large atom sites, which induces a significant distortion to the structure units. The intensity in reciprocal space displays a substantial amount of diffuse scattering with anisotropic distribution, located around the strong Bragg peaks, that can be fully interpreted as resulting from phason fluctuations, with a ratio of the phason elastic constants $\mathbf{K} 2 / \mathbf{K} 1=-0.53$, i.e. close to a three-fold instability limit. This induces a relatively large perpendicular (or phason) Debye-Waller factor, which explains the vanishing of "high-Qperp" reflections.

Keywords: quasicrystals, structure, phasons
MS25-04 The oxidic two-dimensional quasicrystal and its approximant: X-ray analysis of the atomic structure

Holger L. Meyerheim ${ }^{1}$, Sanjiev K. Nayak ${ }^{2}$, Rene Hammer ${ }^{2}$, Sumalay Roy ${ }^{1}$, Waheed Adeagbo ${ }^{2}$, Stefan Förster ${ }^{2}$, Katayoon Mohseni ${ }^{1}$, Wolfram Hergert ${ }^{2}$, Wolf Widdra ${ }^{1,2}$

1. Max-Planck-Institut f. Mikrostrukturphysik, Weinberg 2, D-06120 Halle/S. (Germany)

2. Institut f. Physik, Martin-Luther-Universität Halle-Wittenberg, D-06099 Halle/S. (Germany)

email: hmeyerhm@mpi-halle.mpg.de

Very recently a new type of quasicrystal has been discovered based on an oxide phase, closely related to barium titanate $\left(\mathrm{BaTiO}_{3}\right)$ [1]. The two-dimensional (2D) oxide quasicrystal ${ }^{3}$ (OQC) and its periodic approximant (AP) were grown in situ under ulta-high vacuum conditions on $\operatorname{Pt}(111)$ and investigated by scanning tunneling microscopy (STM) which provided clear evidence for the presence of different tilings. For both, the OQC and the AP only one kind of protrusion could be observed by STM which are arranged in a 2D pattern identified as Stampfli-Gähler tiling [2,3] for the OQC and the Kepler tiling [4] for the AP, respectively. The tiling of the $\mathrm{AP}$ is composed of squares and triangles while in the OQC also rhombs exist. The tilings are fundamental to distinguish between the OQC and the AP. Despite the precise analysis of the tilings, their metric and the approximate stoichiometry of the phases, no detailed structural information is available on the atomic scale. For instance, a straightforward assignment of the protrusions observed in STM to one of the atomic species ( $\mathrm{Ba}, \mathrm{Ti}, \mathrm{O}, \mathrm{Pt})$ is not possible. To this end we have carried out a surface $\mathrm{x}$-ray diffraction study to elucidate the geometric structure of the AP which also allows to develop a model for the OQC structure.

Figure 1 shows the z-projected charge density contour plot calculated on the basis of 44 superlattice reflections. Four unit cells with lattice parameters $a=13.1 \AA, b=12.9$ $\AA, \gamma=90.5^{\circ}$ are shown. Superimposed are the approximate atomic positions derived from the least squares fit $(\mathrm{R}=13 \%)$. We find that the protrusions observed by STM are related to titanium atoms (blue) which are sourrounded by three oxygen atoms approximately forming a triangle. Furthermore, barium atoms (yellow) are located at the origin of the unit cell (plane group p2). Further barium atoms are located in the vicinity of two out of four edges of the squares (triangles) leading to $\mathrm{BaTiO}_{3}$ stoichiometry if no vacancies are present. The relation of the AP to the OQC structure will be discussed.

[1] S. Förster, K. Meinel, R. Hammer, M. Trautmann, and W. Widdra, Nature 502, 215 (2013)

[2] P. Stampfli, Helv. Phys. 59, 1260 (1986)

[3] F. Gähler in: Quasicrystalline Materials (World Scientific, 1988)

[4] J. Kepler: Harmonices Mundi (1619)

The authors acknowledge support and hospitality of the ESRF staff during their stay in Grenoble. This work is supported by the Deutsche Forschungsgemeinschaft (DFG) through SFB 762. 


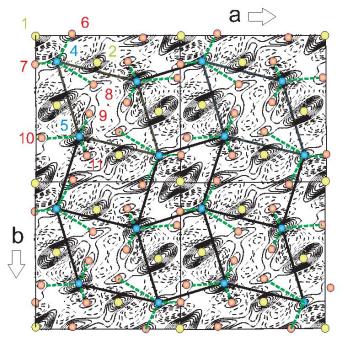

Figure 1. Z-projected charge density contour plot $\rho(x, y)$ of the approximant structure showing four unit cells. The Kepler tiling is indicated by the solid black lines.

Keywords: Oxide Quasicrystal, Approximant, Surface x-ray diffraction, Bariumtitanate

\section{MS25-05 Z-module dislocations in complex intermetallic phases}

Abdullah Sirindil ${ }^{1}$, Loïc Perrière ${ }^{2}$, Sylvie Lartigue-Korinek ${ }^{2}$, Marianne Quiquandon ${ }^{1}$, Richard Portier $^{1}$, Denis Gratias ${ }^{1}$

1. IRCP Chimie-Paristech, 11 rue Pierre et Marie Curie, 75005 Paris FRANCE

2. ICMPE - CNRS 2-8, rue Henri Dunant - 94320 THIAIS-FRANCE Thiais, France

email: abdullah.sirindil@chimie-paristech.fr

Several intermetallic phases have structures where the atoms are located on the sites of a, i.e. at positions $x_{i}$ that are linear integer combination of $\mathrm{N}>\mathrm{d}$ vectors $e$ arithmetically independant, where $d$ is the dimension of the physical space:

$$
x_{i}=\operatorname{Sum}_{\mathrm{k}=1, \mathrm{~N}}\left(n_{k}{ }^{i} e_{i}\right)
$$

These structures are best analyzed as cuts of large periodic objects in a space of dimension $N>d$. These objects can carry high dimensionnal $\mathrm{N}$-dim dislocations the Burgers vectors of which are linear integer combination of the basic vectors $e_{k}$.

We shall illustrate on simples examples the basic properties of these Z-module dislocations also designated by metadislocations, as a function of the relative values of the rank $N$ of the module and the dimension $d$ of the physical space. In particular, when Z-module superabundant, it is possible to build original dislocations the Burgers vector of which is enterly perpendicular to the physical space. These metadislocations are therefore insensitive to any stress fields and behave thus as "scalar" as we shall denominate them. These new defects will be examplified in simple low dimensional cases.

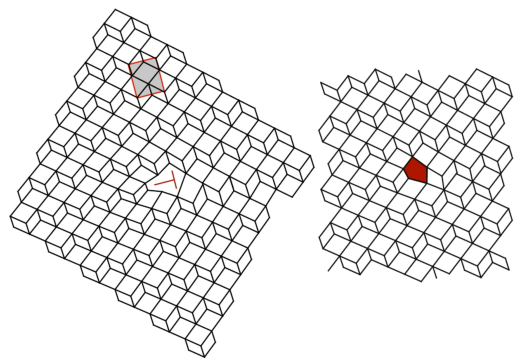

Figure 1. Example of a $2 \mathrm{D}$ periodic structure based on the $\mathrm{Z}^{3}$ : on the left a classical perfect dislocation; on the right, a so-called metadislocation (that is a perfect dislocation at 3D), boarding kind of a stacking fault.

Keywords: approximants, Z-modules, dislocations 